\title{
Accuracy of Density Functional Theory in Prediction of Carbon Dioxide Adsorbent Materials
}

\author{
Claudio Cazorla ${ }^{1}$ and Stephen A. Shevlin ${ }^{2}$ \\ ${ }^{1}$ Institut de Ciència de Materials de Barcelona (ICMAB-CSIC), 08193 Bellaterra, Spain \\ ${ }^{2}$ Department of Chemistry, University College London, London WC1H 0AH, United Kingdon*
}

\begin{abstract}
Density functional theory (DFT) has become the computational method of choice for modeling and characterization of carbon dioxide adsorbents, a broad family of materials which at present are urgently sought after for environmental applications. The description of polar carbon dioxide $\left(\mathrm{CO}_{2}\right)$ molecules in low-coordinated environments like surfaces and porous materials, however, may be challenging for local and semilocal DFT approximations. Here, we present a thorough computational study in which the accuracy of DFT methods in describing the interactions of $\mathrm{CO}_{2}$ with model alkali-earth-metal (AEM, Ca and $\mathrm{Li}$ ) decorated carbon structures, namely anthracene $\left(\mathrm{C}_{14} \mathrm{H}_{10}\right)$ molecules, is assessed. We find that gas-adsorption energies and equilibrium structures obtained with standard (i.e. LDA and GGA), hybrid (i.e. PBE0 and B3LYP) and van der Waals exchange-correlation functionals of DFT dramatically differ from results obtained with second-order Møller-Plesset perturbation theory (MP2), an accurate computational quantum chemistry method. The major disagreements found can be mostly rationalized in terms of electron correlation errors that lead to wrong charge-transfer and electrostatic Coulomb interactions between $\mathrm{CO}_{2}$ and AEMdecorated anthracene molecules. Nevertheless, we show that when the concentration of AEM atoms in anthracene is tuned to resemble as closely as possible to the electronic structure of AEM-decorated graphene (i.e. an extended two-dimensional material), hybrid exchange-correlation DFT and MP2 methods quantitatively provide similar results.
\end{abstract}

PACS numbers: 68.43.Bc, 73.63.Fg, 81.05.U-, 88.05.Np

\section{INTRODUCTION}

The concentration of carbon dioxide $\left(\mathrm{CO}_{2}\right)$ in the atmosphere has increased by about $30 \%$ in the last 50 years and is likely to double over the next few decades as a consequence of fossil-fuel burning for energy generation [1, 2]. This excess of $\mathrm{CO}_{2}$ greenhouse gas may have dramatic negative repercussions on Earth's air quality and climate evolution. Besides exploitation of renewable energy resources, carbon capture and sequestration (CCS) implemented in fossil-fuel energy plants and ambient air have been envisaged as promising cost-effective routes to mitigate $\mathrm{CO}_{2}$ emissions [1, 2]. To this end, membranes and solid sorbents (e.g. activated carbons -AC-, hydrotalcites, and coordination polymers -i.e. zeolitic imidazolate and metal organic frameworks-) are widely considered as the pillars of next-generation CCS technologies because of a encouraging compromise between large gas-uptake capacities, robust thermodynamic stability, fast adsorption-desorption kinetics, and affordable production costs [3]. A key aspect for the success of these materials is to find the optimal chemistry and pore topologies to work under specific thermodynamic conditions [4-6]. Unfortunately, due to the tremendous variety of possible compositions and structures, systematic experimental searches of this kind generally turn out to be cumbersome. In this context, first-principles and classical atomistic simulation approaches emerge as in-

*Electronic address: ccazorla@icmab.es

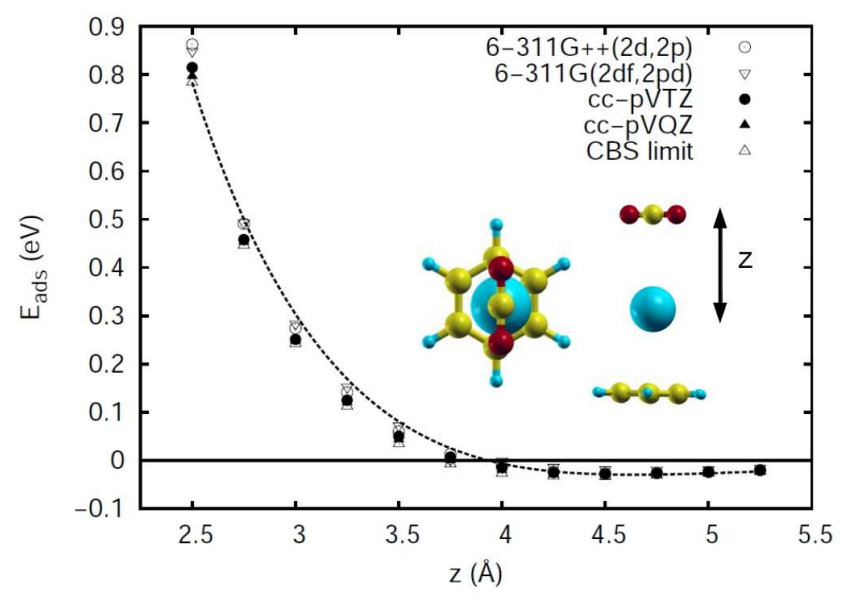

FIG. 1: MP2 adsorption energy results obtained for a small $\mathrm{CO}_{2} / \mathrm{Ca}$-benzene system and expressed as a function of the intermolecular distance $z$ and $\mathrm{AO}$ basis set. $\mathrm{Ca}, \mathrm{C}, \mathrm{H}$, and $\mathrm{O}$ atoms are represented with large blue, yellow, small blue and red spheres, and the dashed line is a guide to the eye.

valuable tools for effective and economical screening of candidate carbon adsorbents.

Density functional theory (DFT) performed with the local-density (LDA) and generalized-gradient (GGA) approximations of the electronic exchange-correlation energy, has become the ab initio method of choice for modeling and characterization of CCS materials. Standard DFT-LDA and DFT-GGA methods are known to describe with precision and affordability a wide spectrum of interactions in bulk crystals and surfaces, however com- 
putational studies on the accuracy of DFT in reproducing $\mathrm{CO}_{2}$-sorbent forces are surprisingly scarce in the literature (see "Methods" section of work [7] and references therein). In view of the ubiquity of DFT methods to CCS science [8 11], it is therefore crucial to start filling this knowledge gap while putting a special emphasis on the underlying physics. Computational benchmark studies on $\mathrm{CO}_{2}$-sorbent interactions, however, are technically intricate and conceptually difficult since most CCS materials have structural motifs that are large in size. In particular, genuinely accurate but computationally very intensive quantum chemistry approaches like MP2 and $\operatorname{CCSD}(\mathrm{T})$ can deal efficiently only with small systems composed of up to few tens of atoms, whereas DFT can be used for much larger systems (i.e. extended -periodically replicated in space- systems composed of up to $1,000-10,000$ atoms). Consequently, computational accuracy tests need to be performed in scaleddown systems resembling to the structure and composition of the material of interest (e.g. the case of organic $\mathrm{C}_{n} \mathrm{H}_{m}$ molecules to graphene $\left.12[15]\right)$. Generalization of so-reached conclusions to realistic systems however may turn out to be fallacious since intrinsic DFT limitations (e.g. exchange self-interaction and electron correlation errors) can be crucial depending, for instance, on the level of quantum confinement imposed by the topology of the system.

In this article, we present the results of a thorough computational study performed on a model system composed of a $\mathrm{CO}_{2}$ molecule and alkali-earth-metal (AEM) decorated anthracene (e.g. $\mathrm{X}-\mathrm{C}_{14} \mathrm{H}_{10}$ with $\mathrm{X}=\mathrm{Ca}, \mathrm{Li}$ ), that consists of standard DFT (i.e. LDA and GGA), hybrid DFT (i.e. PBE0 and B3LYP), van der Waals DFT ( $\mathrm{vdW})$, and MP2 adsorption energy, $\mathrm{E}_{\mathrm{ads}}$ [16], and geometry optimization calculations. It is worth noticing that anthracene is structurally and chemically analogous to the organic bridging ligands found in metal- and covalentorganic frameworks, -MOF and COF-, thus our model conforms to a good representation of a promising class of CCS materials [7, 17-20]. We find that standard, hybrid and vdW functionals of DFT dramatically fail at reproducing $\mathrm{CO}_{2} / \mathrm{AEM}-\mathrm{C}_{14} \mathrm{H}_{10}$ interactions as evidenced by $\mathrm{E}_{\mathrm{ads}}$ discrepancies of $\sim 1-2 \mathrm{eV}$ found with respect to MP2 calculations. This failure is mainly due to electron correlation errors that lead to inaccurate electron charge transfers and exaggerated electrostatic Coulomb interactions between $\mathrm{CO}_{2}$ and $\mathrm{X}-\mathrm{C}_{14} \mathrm{H}_{10}$ molecules. In the second part of our study we analyse whether our initial conclusions can be generalized or not to extended carbon-based materials, another encouraging family of $\mathrm{CO}_{2}$ sorbents 21 24]. For this, we tune the concentration of calcium atoms in anthracene so that the partial density of electronic states (pDOS) of the model system resembles as closely as possible to the pDOS of Cadecorated graphene. In this case we find that DFT and MP2 methods qualitatively provide similar results, with hybrid DFT and MP2 in almost quantitative agreement.

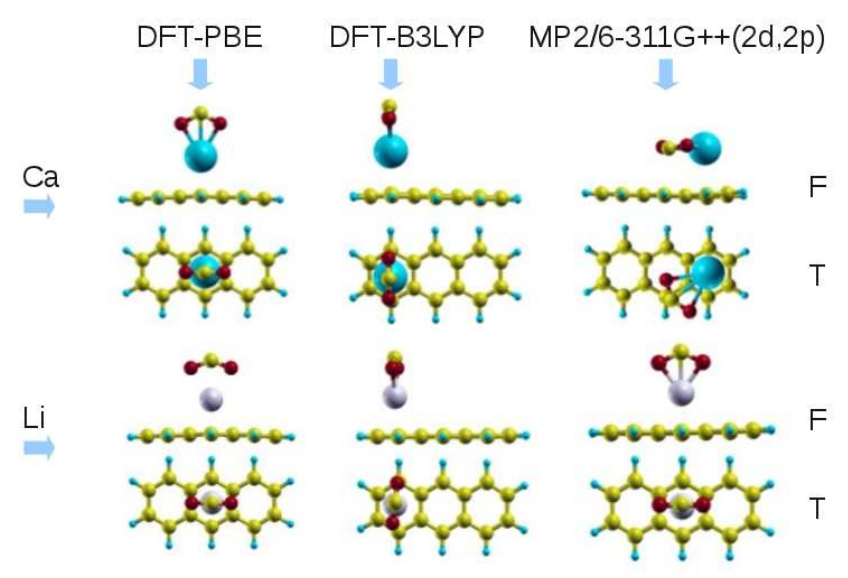

FIG. 2: Front (F) and top (T) views of equilibrium $\mathrm{CO}_{2}$ adsorption structures in $\mathrm{Ca}$ - and Li-anthracene as obtained with standard and hybrid DFT and MP2 methods. Li atoms are represented with purple spheres.

\section{COMPUTATIONAL METHODS}

Standard DFT calculations were done using the plane wave code VASP [25, 26] while hybrid DFT and MP2 results were obtained with the atomic orbitals $(\mathrm{AO})$ code NWCHEM [27]. Numerical consistency between the two codes was checked at the DFT-PBE energy level. The value of all technical parameters were set in order to guarantee convergence of the total energy to less than $1 \mathrm{meV} /$ atom. Optimized structures were determined by imposing an atomic force tolerance of $0.01 \mathrm{eV} / \AA$ and verified as minima on the potential energy surface by vibrational frequency analysis. Basis-set superposition errors (BSSE) in hybrid DFT and MP2 energy calculations were corrected using the counter-poise recipe [28]. Indeed, only results obtained in the complete-basis-set (CBS) limit [29, 30] can be regarded as totally BSSE free however reaching that limit in our calculations turned out to be computationally prohibitive due to the size of the systems and large number of cases considered. Nevertheless, we checked in a reduced Ca-benzene system that MP2 binding energy results obtained with large Dunninglike $\mathrm{AO}$ basis sets (i.e. triple zeta cc-pVTZ and quadruple zeta cc-pVQZ) and in the CBS limit differed at most by $20 \mathrm{meV}$ (see Fig. 1), thus we assumed the MP2/cc-pVTZ method to be accurate enough for present purporses (i.e. as it will be shown later, the reported discrepancies are in the order of $1-2 \mathrm{eV}$ ) and regarded it as "gold standard". It is worth noting that MP2 results obtained with medium and large Pople-like AO basis sets (i.e. 6$311 \mathrm{G}++(2 \mathrm{~d}, 2 \mathrm{p})$ and $6-311 \mathrm{G}(2 \mathrm{df}, 2 \mathrm{pd}))$ are also in notable agreement with MP2/cc-pVTZ results (i.e. $\mathrm{E}_{\text {ads }}$ differences of $20-30 \mathrm{meV}$ in the worse case) whereas MP2/6$31 \mathrm{G}++$ estimations (not shown in the figure) turn out to be not so accurate. 

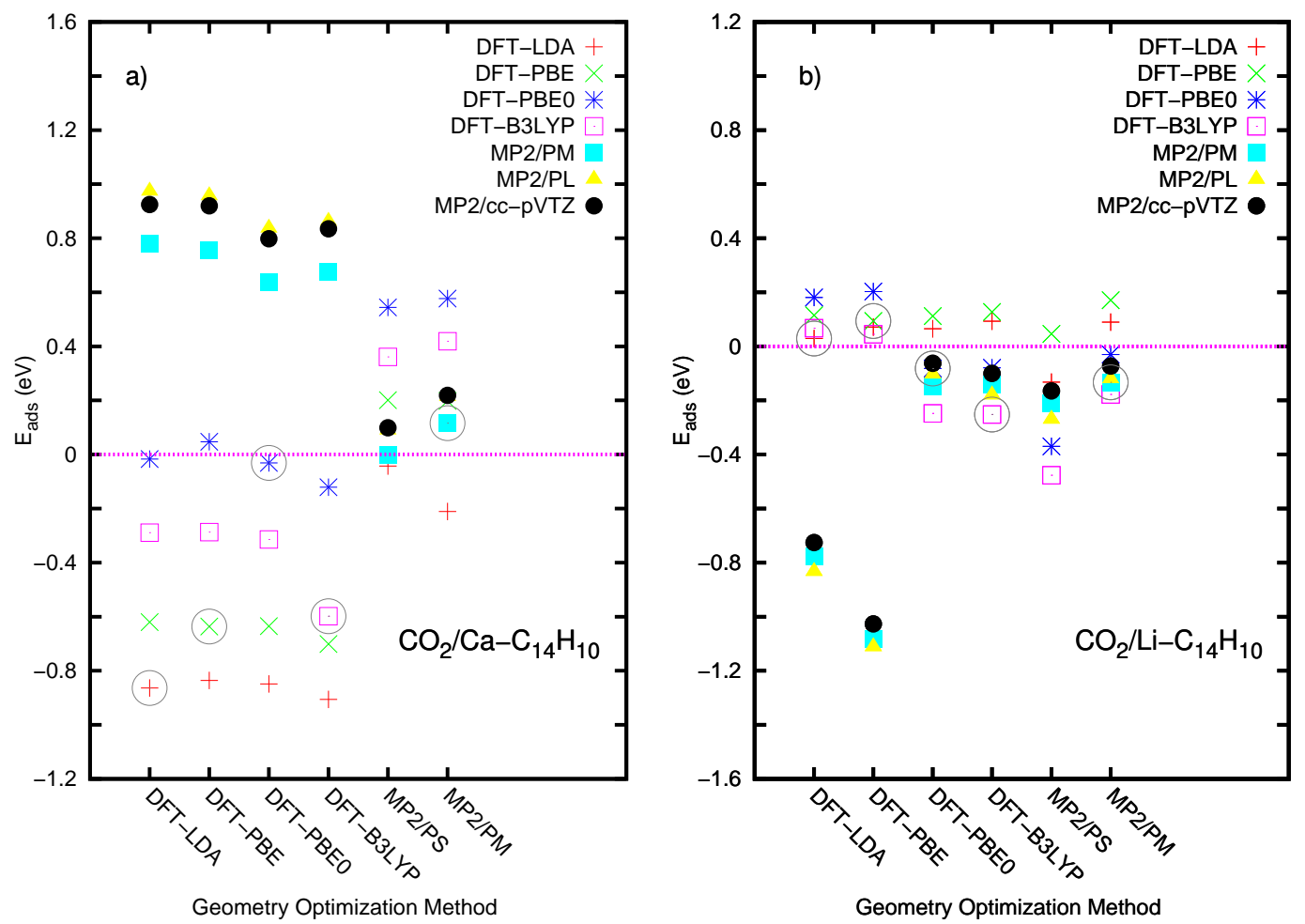

FIG. 3: $\mathrm{CO}_{2}$-adsorption energy results obtained for Ca- (a) and Li-anthracene (b), using different optimization and evaluation methods. Cases in which optimization and evaluation methods coincide are highlighted with large grey circles. PS, PM, and PL notation stands for 6-31G++, 6-311G++ $(2 \mathrm{~d}, 2 \mathrm{p})$, and 6-311G(2df,2pd) Pople AO basis sets, respectively.

\section{RESULTS}

\section{A. AEM-decorated anthracene systems}

Carbon dioxide adsorption energy and equilibrium geometry results obtained for AEM-anthracene systems are shown in Figs. 2 and 3. Since the number of reaction coordinates in $\mathrm{CO}_{2} / \mathrm{AEM}$-anthracene systems (i.e. intermolecular distances and molecular bond and torsional angles) is considerably large, rather than parameterizing $E_{\text {ads }}$ curves as a function of just few of them, first we performed DFT and MP2 atomic relaxations and then calculated all DFT and MP2 energies in the resulting equilibrium structures. In doing this, we disregard local minimum conformations, as customarily done in computational materials studies, and gain valuable insight into the energy landscape provided by each potential. It is important to note that due to the size of the systems considered we were able to perform tight MP2 atomic relaxations only at the $6-31 \mathrm{G}++$ and $6-311 \mathrm{G}++(2 \mathrm{~d}, 2 \mathrm{p})$ levels . Nevertheless, MP2/6-311G++ $(2 \mathrm{~d}, 2 \mathrm{p})$ and benchmark MP2/cc-pVTZ equilibrium structures are very likely to be equivalent since results obtained with both methods are in fairly good agreement (see Figs. 1 and 3), and $\mathrm{MP} 2 / 6-31 \mathrm{G}++$ and $\mathrm{MP} 2 / 6-311 \mathrm{G}++(2 \mathrm{~d}, 2 \mathrm{p})$ equilibrium geometries are already very similar (the former case not shown here).
First, we note that equilibrium DFT and MP2 structures obtained for both Ca- and Li-anthracene systems are perceptibly different (see Fig. 2). Of particular concern is the Ca-anthracene system where, depending on the geometry optimization method used, the plane containing the $\mathrm{CO}_{2}$ molecule orientates perpendicularly (DFT) or parallel (MP2) to anthracene. Also, we observe important differences between DFT-PBE and DFTB3LYP optimized geometries, the Ca atom being displaced towards an outside carbon ring in the hybrid case (as it also occurs in the MP2-optimized system). Structural differences among Li-anthracene systems are similar to those already explained except that the gas molecule always binds on top of the Li atom and no off-center AEM shift appears in MP2 optimizations.

Concerning $\mathrm{E}_{\text {ads }}$ results (see Fig. 3), let us concentrate first on the $\mathrm{Ca}-\mathrm{C}_{14} \mathrm{H}_{10}$ case. As one can see, adsorption energies calculated with the same evaluation and geometry optimization method (highlighted with large grey circles in the figure) are very different. In particular, DFT methods always predict thermodynamically favorable $\mathrm{CO}_{2}$-binding to Ca-anthracene, with DFT-LDA and DFT-PBE0 providing the largest and smallest $\mathrm{E}_{\text {ads }}$ values, whereas MP2/6-311G $++(2 \mathrm{~d}, 2 \mathrm{p})$ calculations show the opposite. Moreover, with MP2/6-311G(2df,2pd) and MP2/cc-pVTZ methods large and positive adsorption energies of $\sim 0.6-1.0 \mathrm{eV}$ are obtained for DFToptimized geometries, in stark contrast to DFT E $\mathrm{E}_{\text {ads }}$ re- 
sults (i.e. $\sim-1.0-0.0 \mathrm{eV}$ ). Adsorption energy disagreements in $\mathrm{Li}_{-} \mathrm{C}_{14} \mathrm{H}_{10}$ systems are not as dramatic as just described, although the performance of standard DFT methods still remains a cause for concern. Specifically, DFT-LDA and DFT-PBE predict unfavorable $\mathrm{CO}_{2}$-binding whereas hybrid DFT and MP2 methods predict the opposite. Also, computed MP2 energies in standard DFT-optimized structures are negative and noticeably larger than DFT $\left|\mathrm{E}_{\mathrm{ads}}\right|$ values. Interestingly, the series of binding energies calculated for hybrid DFT and MP2/6-311G++ $(2 \mathrm{~d}, 2 \mathrm{p})$ geometries are in remarkably good agreement in spite of the evident structural differences involved (see Fig. 2).

Since the agreement between MP2 and hybrid DFT results in $\mathrm{Ca}-\mathrm{C}_{14} \mathrm{H}_{10}$ is only marginally better than achieved with LDA or GGA functionals, common selfinteraction exchange errors alone cannot be at the root of standard DFT failure. Consequently, DFT difficulties at fully grasping electron correlations, which in the studied complexes account for the $44 \%$ to $57 \%$ of the total binding energy, must be the major factor behind the discrepancy. In fact, upon gas-adsorption important dispersive dipole-dipole and dipole-quadrupole forces appear in the systems as a consequence of $\mathrm{CO}_{2}$ inversion symmetry breaking (i.e. the polar molecule is bent) which cannot be reproduced by either local, semilocal or hybrid DFT approximations. Moreover, the non-linearity of the gas molecule also indicates the presence of large electron transfers which are well-known to pose a challenge for description to standard and hybrid DFT methods [31]. But, which of these DFT shortcommings, i.e. omission of non-local interactions, charge-transfer errors or a mix of both, is the predominant factor behind $\mathrm{E}_{\text {ads }}$ inaccuracies? In order to get insight into this question, we performed frontier molecular orbital and charge distribution analysis on isolated and joint $\mathrm{Ca}-\mathrm{C}_{14} \mathrm{H}_{10}$ and $\mathrm{CO}_{2}$ complexes [32]. We found that the energy difference between the HOMO of Ca-anthracene and the LUMO of the gas molecule, $\Delta E_{\text {front }}$, varied from $0.7-0.9 \mathrm{eV}$ to $-1.4 \mathrm{eV}$ when either calculated with DFT (standard and hybrid) or MP2/cc-pVTZ methods. Positive and large $\Delta E_{\text {front }}$ values do imply large reactivity and charge transfers from Ca- $\mathrm{C}_{14} \mathrm{H}_{10}$ to $\mathrm{CO}_{2}$ molecules, $\Delta Q$. In fact, this is consistent with results shown in Fig. 3 and also with our charge distribution analysis performed: $\Delta Q$ values obtained with DFT amount to $\sim 1 e^{-}$, about a $50 \%$ larger than computed with MP2/cc-pVTZ [33]. Standard and hybrid DFT approximations, therefore, provide overly charged donor $(+)$ and acceptor (-) species which in the joint complexes are artificially stabilized by the action of exaggerated electrostatic interactions. In view of this finding, and also of the large size of the binding energies reported, we tentatively identify the inability of DFT methods to correctly describe charge-transfer interactions (and not the omission of non-local interactions) as the principal cause behind its failure. Calculations done on Li-anthracene systems appear to support this hypothesis since a mild improvement on the agreement between hybrid DFT and MP2 methods is obtained (not seen in the standard cases) which is accompanied by smaller $\Delta E_{\text {front }}$ discrepancies (i.e. of just few tenths of an eV). Also, the amount of electronic charge transferred from Li- $\mathrm{C}_{14} \mathrm{H}_{10}$ to $\mathrm{CO}_{2}$ does not change significantly when either calculated with hybrid DFT or MP2 methods (i.e. 0.6 and $0.4 e^{-}$, respectively).

In order to fully quantify the effect of neglecting nonlocal interactions and their role on standard and hybrid DFT $E_{\text {ads }}$ inaccuracies, we conducted additional energy calculations using two different DFT van der Waals approaches implemented in VASP. The first of these methods corresponds to that developed by Grime, also known as DFT-D2 [34], in which a simple pair-wise dispersion potential is added to the conventional Kohn-Sham DFT energy. The second approach, referred to as DFT-vdW here, is based on Dion et al.'s proposal [35 37] for which a non-local correlation functional is explicitly constructed. We considered three different equilibrium configurations (i.e. those obtained in DFT-PBE, DFT-B3LYP and MP2/cc-pVTZ geometry optimizations) and calculated the corresponding DFT-D2 and DFT-vdW adsorption energies. In Table I, we report the results of these calculations. As one can see, the overall effect of considering non-local interactions is to decrease $\mathrm{E}_{\mathrm{ads}}$ values by about few tenths of an $\mathrm{eV}$, increasing so slightly the discrepancies with respect to the MP2/cc-pVTZ method. Also, we find that van der Waals corrections obtained with both DFT-D2 and DFT-vdW methods are fairly similar. For instance, $\Delta E_{\mathrm{D} 2}$ and $\Delta E_{\mathrm{vdW}}$ differences (taken with respect to DFT-PBE values, see Table I) computed in the DFT-B3LYP case amount to -0.08 and $-0.13 \mathrm{eV}$, respectively. We also found that the equilibrium $\mathrm{CO}_{2} / \mathrm{Ca}-$ $\mathrm{C}_{14} \mathrm{H}_{10}$ geometry determined with the DFT-D2 method is practically identical to that found with DFT-PBE. Therefore, our above assumption on the causes behind unsatisfactory description of specific CCS processes by DFT methods (i.e. charge-transfer interaction errors) turns out to be rigorously demonstrated.

Overall, the results presented in this section show the key importance of charge-transfer interactions in AEMbased CCS nanoparticles which, as we mentioned in the Introduction, can be reasonably generalized to similar covalent organic structures. Consequently, MP2 or other efficient computational schemes embodying also manyelectron correlations (see for instance Ref. 38]) must be employed for the rational design and characterization of these complexes.

\section{B. Emulating extended AEM-decorated carbon surfaces}

In view of the composition and structure of anthracene, it appears tempting to generalize our previous conclusions to extended carbon-based materials (e.g. AC and nanostructures). Nevertheless, we show in Fig. 4 that Ca-anthracene and Ca-graphene are quite distinct sys- 


\begin{tabular}{cccc}
\hline \hline & \multicolumn{2}{c}{ Geometry Optimization Method } \\
& & & \\
\cline { 2 - 3 } & $\mathrm{DFT}-\mathrm{PBE}$ & $\mathrm{DFT}-\mathrm{B} 3 \mathrm{LYP}$ & $\mathrm{MP} 2 / \mathrm{PM}$ \\
\hline $\mathrm{DFT}-\mathrm{D} 2$ & -0.719 & -0.775 & -0.056 \\
$\left(\Delta \mathrm{E}_{\mathrm{D} 2}\right)$ & $(-0.083)$ & $(-0.074)$ & $(-0.254)$ \\
$\mathrm{DFT}-\mathrm{vdW}$ & -0.856 & -0.836 & -0.115 \\
$\left(\Delta \mathrm{E}_{\mathrm{vd}}\right)$ & $(-0.220)$ & $(-0.135)$ & $(-0.313)$ \\
$\mathrm{MP} 2 / \mathrm{cc}-\mathrm{pVTZ}$ & 0.920 & 0.835 & 0.219 \\
\hline \hline
\end{tabular}

TABLE I: $\mathrm{CO}_{2}$-adsorption energy results obtained for Ca-anthracene using the DFT-D2 34], DFT-vdW 35 37], and (for comparison) MP2/cc-pVTZ methods. $\Delta \mathrm{E}_{\mathrm{D} 2}$ values correspond to $\mathrm{E}_{\mathrm{DFT}-\mathrm{D} 2}-\mathrm{E}_{\mathrm{DFT}-\mathrm{PBE}}$ energy differences, and $\Delta \mathrm{E}_{\mathrm{vdW}}$ to $\mathrm{E}_{\mathrm{DFT}-\mathrm{vdW}}-\mathrm{E}_{\mathrm{DFT}-\mathrm{PBE}}$. All energies are expressed in units of $\mathrm{eV}$.

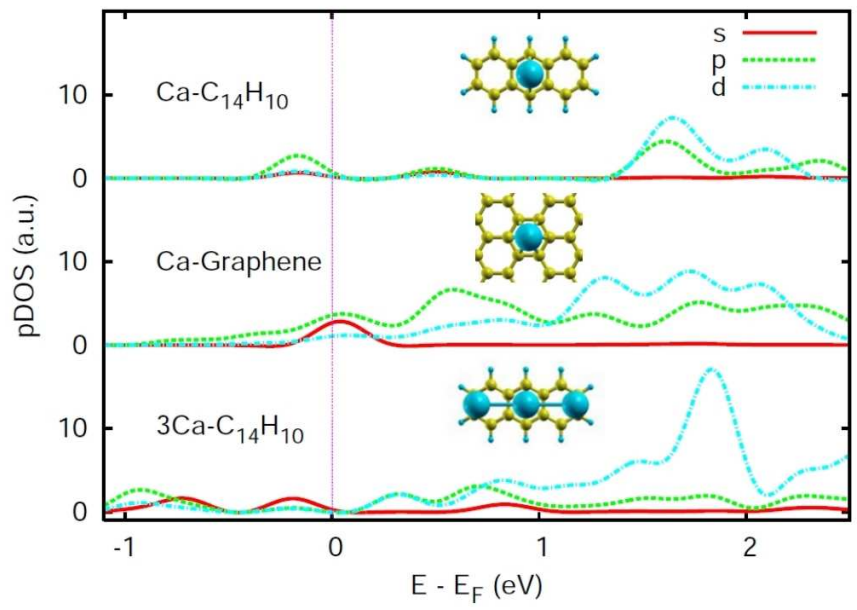

FIG. 4: Partial density of electronic states (pDOS) obtained for Ca-anthracene, Ca-graphene, and 3Ca-anthracene using the DFT-PBE method. In each case, energies have been shifted to the corresponding Fermi level (i.e. $-3.02,-0.93$, and $-2.73 \mathrm{eV}$, respectively).

tems in terms of electronic structure since partially occupied $s$ and $d$ electron orbitals are missing in the former. It is important to note that equivalent partial density of states (pDOS) dissimilarities are also found when larger $\mathrm{C}_{n} \mathrm{H}_{m}$ molecules are considered (e.g. the case of Cacoronene [14]). In consequence of these differences, hybridization between $s d$-sorbent and $p-\mathrm{CO}_{2}$ orbitals leading to strong gas attraction will be more limited in Ca$\mathrm{C}_{14} \mathrm{H}_{10}$ complexes than in Ca-decorated graphene [21].

Aimed at improving the likeness among the pDOS of the model system and Ca-decorated graphene while constraining the size of the former system to be small, we increased the number of $\mathrm{Ca}$ atoms decorating anthracene [21, 39]. We found that pDOS features in 3Ca-

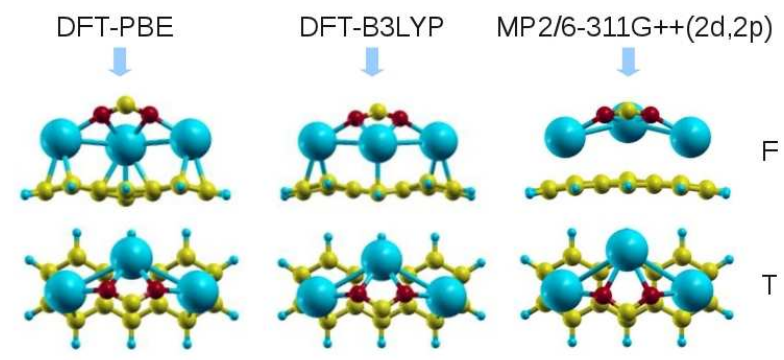

FIG. 5: Front (F) and top (T) views of equilibrium $\mathrm{CO}_{2}$ adsorption structures in $3 \mathrm{Ca}$-anthracene as obtained with standard and hybrid DFT and MP2 methods. The atomic color code is the same than used in previous figures.

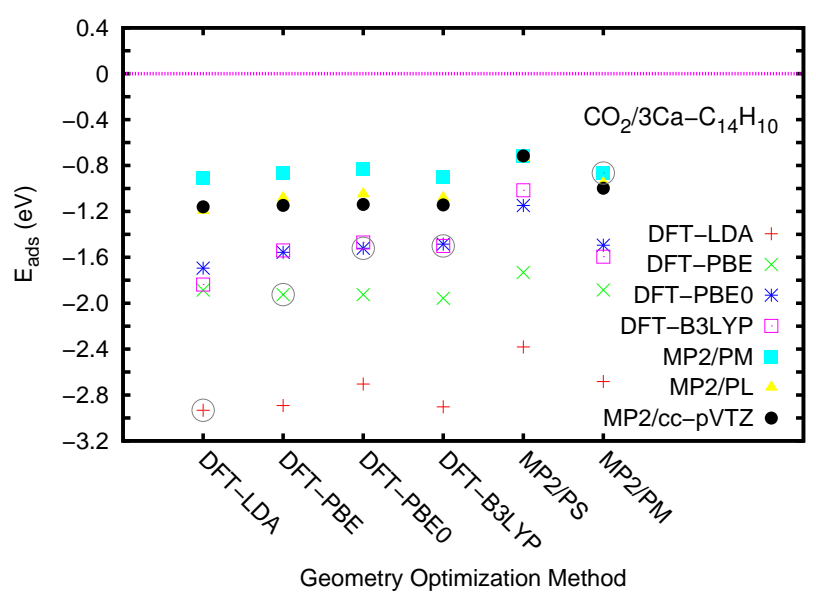

FIG. 6: $\mathrm{CO}_{2}$-adsorption energy results obtained for $3 \mathrm{Ca}$ anthracene using different optimization and evaluation methods. Cases in which optimization and evaluation methods coincide are highlighted with large grey circles. PS, PM, and PL notation stands for $6-31 \mathrm{G}++, 6-311 \mathrm{G}++(2 \mathrm{~d}, 2 \mathrm{p})$, and $6-$ $311 \mathrm{G}(2 \mathrm{df}, 2 \mathrm{pd})$ Pople AO basis sets, respectively. 
$\mathrm{C}_{14} \mathrm{H}_{10}$ are already compatible with those relevant for $\mathrm{CO}_{2}$-binding to Ca-graphene (see Fig. (4) thus, in spite of the obvious chemical and structural deformations introduced (e.g. now the anthracene molecule is significantly bent, see Fig. 5), we performed additional benchmark calculations for this system.

In Figs. 5 and 6 we show the resulting optimized geometries and $\mathrm{E}_{\text {ads }}$ values. As can be appreciated standard DFT, hybrid DFT and MP2 calculations are now in qualitative good agreement: all methods predict equivalent equilibrium structures and thermodynamically favorable $\mathrm{CO}_{2}$-binding. Moreover, adsorption energy differences between hybrid DFT and MP2 methods amount to less than $0.4 \mathrm{eV}$ in most studied geometries with hybrid DFT systematically providing the smaller values. Therefore, the agreement between hybrid DFT and MP2 approaches can be regarded in this case as almost quantitative. On the other hand, standard DFT approximations tend to significantly overestimate $\mathrm{E}_{\text {ads }}$ (i.e. by about $\sim 1.0-2.0 \mathrm{eV}$ with respect to the MP2/ccpVTZ method). It must be stressed that charge-transfer interactions in Ca-overdecorated organic complexes are very intense and play also a dominant role. Indeed, the amount of electron charge transferred from $3 \mathrm{Ca}-\mathrm{C}_{14} \mathrm{H}_{10}$ to $\mathrm{CO}_{2}$ is $\Delta Q=1.3 e^{-}$according to the MP2/cc-pVTZ method and also hybrid DFT approximations. In contrast, standard DFT methods predict exceedingly large $\Delta Q$ values of $\sim 1.6-1.8 e^{-}$. We identify therefore self-interaction exchange errors, which now appear as a consequence of populating spatially localized $d$-Ca orbitals [21, 40], as the principal cause for the overestimation of $\mathrm{CO}_{2}$-binding by standard DFT approaches.

In the light of the results presented in this section, we conclude that standard DFT modeling of extended carbon-based CCS materials can be expected to be correct only at the qualitative level. On the other hand, hybrid DFT approximations conform to a wellbalanced representation of the relevant interactions in AEM-decorated carbon surfaces thus we propose using them when pursuing accurate description of these sys- tems.

\section{CONCLUSIONS}

We have performed a thorough computational study in which the failure of standard, hybrid and van der Waals DFT methods at describing the interactions between $\mathrm{X}$-anthracene $(\mathrm{X}=\mathrm{Li}$ and $\mathrm{Ca})$ and $\mathrm{CO}_{2}$ molecules is demonstrated. The origins of this deficiency mainly resides on the inability of standard and hybrid DFT approximations to correctly describe charge-transfer interactions. This finding has major implications in modeling and characterization of coordination polymer frameworks (e.g. MOF and $\mathrm{COF}$ ) with applications in carbon capture and sequestration. As an effective strategy to get rid of these computational shortcomings, we propose using MP2 or other effective computational approaches incorporating many-electron correlations. Moreover, based on the similarities in electronic structure found between Cagraphene and $3 \mathrm{Ca}-\mathrm{C}_{14} \mathrm{H}_{10}$ systems (and in spite of their obvious chemical and structural differences) and the tests performed, we argue that standard DFT modeling of extended carbon-based materials may be expected to be correct at the qualitative level. On the other hand, hybrid DFT approximations will provide quantitative information on these systems. The conclusions presented in this work suggest revision of an important number of computational studies that are relevant to CCS materials engineering.

\section{Acknowledgments}

This work was supported by MICINN-Spain (Grants No. MAT2010-18113, CSD2007-00041, and FIS200803845) and computing time was kindly provided by CESGA.
[1] B. Metz, O. Davidson, H. de Coninck, M. Loos, and L. Meyer, Intergovernmental Panel on Climate Change (IPCC) Special Report on Carbon Dioxide Capture and Storage, Cambridge University Press, New York, 3-15 (2005).

[2] N. Nakicenovic and R. Swart, Intergovernmental Panel on Climate Change (IPCC) Special Report on Emissions Scenarios, Cambridge University Press, U.K., 570 (2000).

[3] D. M. Alessandro, B. Smit, and J. R. Long, Angew. Chem. Int. Ed. 49, 6058 (2010).

[4] Y.-S. Bae, O. K. Farha, A. M. Spokoyny, C. A. Mirkin, J. T. Hupp, and R. Q. Snurr, Chem. Comm. 35, 4135 (2008).

[5] N. Hedin, L. Chen, and A. Laaksonen, Nanoscale 2, 1819 (2010).

[6] A. C. Sudik, A. R. Millward, N. W. Ockwig, A. P. Cote,
J. Kim, and O. M. Yaghi, JACS 127, 7110 (2005).

[7] A. Torrisi, C. Mellot-Draznieks, and R. G. Bell, J. Chem. Phys. 130, 194703 (2009).

[8] N. Kumar, K. S. Subrahmanyam, P. Chaturbedy, K. Raidongia, A. Govindaraj, K. P. S. S. Hembram, A. K. Mishra, U. V. Waghmare, and C. N. R. Rao, ChemSusChem 4, 1662 (2011).

[9] Y. Duan, D. R. Luebke, H. W. Pennline, B. Li, M. J. Janik, and J. Woods Halley, J. Phys. Chem. C 116, 14461 (2012).

[10] Y. Jiao, A. Du, Z. Zhu, V. Rudolph, and Sean C. Smith, J. Phys. Chem. C 114, 7846 (2010).

[11] Z. Xiang, D. Cao, J. Lan, W. Wanga and D. P. Broom, Energy Environ. Sci. 3, 1469 (2010).

[12] J. Cha et al., Phys. Rev. Lett. 103, 216102 (2009).

[13] Y. Ohk, Y.-H. Kim, and Y. Jung, Phys. Rev. Lett. 104, 
179601 (2010).

[14] C. Cazorla, S. A. Shevlin, and Z. X. Guo, Phys. Rev. B 82, 155454 (2010).

[15] C. Cazorla, Thin Solid Films 518, 6951 (2010).

[16] $\mathrm{E}_{\text {ads }}$ is defined as the energy difference between the fully relaxed $\mathrm{CO}_{2} / \mathrm{X}$-anthracene system and the individually optimized $\mathrm{CO}_{2}$ and $\mathrm{X}$-anthracene components. Negative (positive) $\mathrm{E}_{\text {ads }}$ values thus indicate thermodynamically favorable or exothermic (unfavorable or endothermic) $\mathrm{CO}_{2}$-binding to $\mathrm{X}$-anthracene.

[17] J. Lan et al., ACS Nano 4, 4225 (2010).

[18] A. Torrisi, R. G. Bell, and C. Mellot-Draznieks, Crystal Growth and Design 10, 2839 (2010).

[19] K. D. Vogiatzis et al., ChemPhysChem 10, 374 (2009).

[20] Y. Yao et al., Phys. Rev. B 85, 064302 (2012).

[21] C. Cazorla, S. A. Shevlin, and Z. X. Guo, J. Phys. Chem. C 115, 10990 (2011).

[22] B. Gao et al., J. Phys. Chem. C 115, 9969 (2011).

[23] A. K. Mishra and S. Ramaprabhu, Energy Environ. Sci. 4, 889 (2011).

[24] A. K. Mishra and S. Ramaprabhu, AIP Advances 1, 032152 (2011).

[25] G. Kresse and J. Furthmüller, Phys. Rev. B 54, 11169 (1996).

[26] We used the "projector augmented wave" method, P. E. Blöchl, Phys. Rev. B 50, 17953 (1994), to represent the ionic cores and considered the following electrons as valence: C's $2 s$ and $2 p$, H's $1 s$, O's $2 s$ and $2 p$, Li's $1 s$ and $2 s$, and Ca's $3 p$ and $4 s$. A large energy cut-off of $700 \mathrm{eV}$ was used in all DFT geometry optimization and energy calculations. The size of the simulation box was $30 \AA \times 30 \AA \times 30 \AA$ and a special Monkhorst-Pack kpoint grid of $2 \times 2 \times 2$ was used for sampling of the first Brillouin zone.

[27] E. J. Bylaska et al., NWChem, A Computational Chemistry Package for Parallel Computers, Version 5.1
(2007).

[28] S. F. Boys and F. Bernardi, Mol. Phys. 19, 553 (1970).

[29] A. Halkier et al., Chem. Phys. Lett. 286, 243 (1998).

[30] A. Halkier et al., Chem. Phys. Lett. 302, 437 (1999).

[31] S. N. Steinmann, C. Piemontesi, A. Delachat, and C. Corminboeuf, J. Chem. Theory Comput. 8, 1629 (2012).

[32] In VASP calculations, we performed charge distribution analysis based on Bader's quantum theory (i.e. Bader, R. G. W. Atoms in Molecules. A Quantum Theory; Oxford University Press: New York, 1990; Henkelman, G.; Arnaldsson, A.; Johnsson, H. Comput. Mater. Sci. 2006, 36, 354). In NWCHEM calculations, we adopted the Mulliken population values already provided by the same code.

[33] Mulliken population and Bader charge analysis are in principle non-equivalent approaches so that direct comparison between results obtained with them may sometimes turn out to be misleading. Nevertheless, we checked at the DFT-PBE level of theory that $\Delta Q$ values predicted with the two methods agree within $10 \%$. This discrepancy is much smaller than the $\Delta Q$ discrepancies of $50 \%$ reported in this study thus our conclusions drawn from Mulliken population and Bader charge comparisons can be considered as fully meaningful.

[34] S. Grimme, J. Comp. Chem. 27, 1787 (2006).

[35] M. Dion, H. Rydberg, E. Schröder, D. C. Langreth, and B. I. Lundqvist, Phys. Rev. Lett. 92, 246401 (2004).

[36] G. Rom'an, J. M. Soler, Phys. Rev. Lett. 103, 096102 (2009).

[37] J. Klimes, D. R. Bowler, and A. Michaelides, J. Phys.: Cond. Matt. 22, 022201 (2010).

[38] L. Schimka et al., Nat. Mater. 9, 741 (2010).

[39] S. L. Sun et al., J. Phys. Chem. B 109, 12868 (2005).

[40] C. Cazorla, V. Rojas-Cervellera, and C. Rovira, J. Mater. Chem. 22, 19684 (2012). 